\title{
Impact of Pseudomonas aeruginosa quorum sensing signaling molecules on adhesion and inflammatory markers in endothelial cells
}

\author{
Carmen Curutiu ${ }^{1,2}$, Florin Iordache ${ }^{\star 3,4}$, Veronica Lazar ${ }^{1,2}$, Aurelia Magdalena Pisoschi $^{3}$, \\ Aneta Pop ${ }^{3}$, Mariana Carmen Chifiriuc ${ }^{1,2}$ and Alina Maria Hoban ${ }^{1,2}$
}

Open Access

\author{
Full Research Paper

\section{Address:} \\ ${ }^{1}$ University of Bucharest, Faculty of Biology, Department of \\ Microbiology-Immunology, Bucharest, Romania, ${ }^{2}$ Research Institute \\ of the University of Bucharest, Romania, ${ }^{3}$ University of Agronomical \\ Sciences and Veterinary Medicine, Faculty of Veterinary Medicine, \\ Bucharest, Romania and ${ }^{4}$ Institute of Cellular Biology and Pathology \\ Nicolae Simionescu of Romanian Academy, Romania \\ Email: \\ Florin lordache ${ }^{*}$ - floriniordache84@yahoo.com \\ * Corresponding author

\section{Keywords:} \\ adhesion; host-pathogen interaction; inflammation; Pseudomonas; \\ quorum sensing
}

Beilstein J. Org. Chem. 2018, 14, 2580-2588. doi:10.3762/bjoc. 14.235

Received: 02 August 2018

Accepted: 19 September 2018

Published: 05 October 2018

This article is part of the thematic issue "Antibacterials, bacterial small molecule interactions and quorum sensing".

Guest Editor: D. Spring

(c) 2018 Curutiu et al.; licensee Beilstein-Institut. License and terms: see end of document.

\begin{abstract}
Pseudomonas aeruginosa relies on the quorum sensing (QS) signaling system as a central regulator mechanism of virulence expression that contributes to the formation and maintenance of biofilms and tolerance to conventional antimicrobials. QS Signaling molecules (QSSMs) may be recognized and may function also within the host cells, being potentially involved in the progression of the infectious process. In this study we evaluate the expression of adhesion and inflammatory molecules in endothelial cells treated with $P$. aeruginosa QSSMs, in order to bring new insights on the mechanisms involved in the interaction of $P$. aeruginosa with host cells during the infectious process. Endothelial cells were stimulated with $20 \mu \mathrm{M}$ of main $P$. aeruginosa QSSMs (OdDHL $=N$-(3oxododecanoyl)-L-homoserine lactone, C4HSL = N-butyryl-L-homoserine lactone, PQS = 2-heptyl-3-hydroxy-4(1H)-quinolone and $H H Q=2$-heptyl-4-quinolone). Adherence to endothelial cells, inert substratum and biofilm formation was evaluated. The expression of adhesion molecules (VE-cadherin, PECAM-1, ICAM-1, and P-selectin) and inflammatory response molecules (IL-1 $\beta$, IL-6, TNF $\alpha$, TGF $\beta$, and eNOS) was assessed by qRT-PCR and flow cytometry. Our results showed that bacterial adherence to inert substratum and biofilm were decreased in the presence of all tested QSSMs. The adherence index of PAO1 laboratory strain to host cells was decreased between $10-40 \%$ in the presence of QSSMs, as compared to untreated control. Expression of eukaryotic cells adhesion molecules ICAM-1 and P-selectin was stimulated by QSSMs, whereas VE-cadherin and PECAM-1 levels were increased only by C4HSL. The inflammatory response of endothelial cells was also modulated, as observed by the modified expression of IL-1 $\beta$ (for C4HSL, PQS and HHQ), IL-6 (for C4HSL and HHQ), TNF $\alpha$ (for C4HSL and HHQ), TGF $\beta$, and eNOS factors. Our
\end{abstract}


results demonstrate that the main pseudomonadal QSSMs differentially modulate endothelial cells adhesion and proinflammatory cytokine expression. These observations provide new insights in the mechanisms by which different QSSMs activate endothelial cells and modulate the infectious process, and support the importance of recent studies aiming to develop anti-QS therapeutic strategies to fight against $P$. aeruginosa infections.

\section{Introduction}

Pseudomonas (P.) aeruginosa is an opportunistic pathogen that causes severe and persistent infections in immune compromised individuals and in patients with bronchiectasis or cystic fibrosis. The infections become chronic, as $P$. aeruginosa develops resistance to conventional antibiotics due to its ability to produce virulence factors and modulate immune defenses by quorum sensing (QS) and biofilm production. Pseudomonas aeruginosa is recognized as the principal pathogen responsible of high morbidity and mortality in patients with cystic fibrosis, one of the most common life-threatening autosomal recessive genetic disease in Northwest European populations, determined by mutations in the cystic fibrosis transmembrane conductance regulator (CFTR) gene [1]. This mutation determines alteration of ion transport and subsequent dehydration of the airway surface liquid, resulting in a viscous mucus layer on the airway surface of cystic fibrosis patients that deteriorate the mucociliary clearance and enhance the infection, inflammation and respiratory insufficiency [2]. P. aeruginosa pathogenesis is multifactorial, as suggested by the large number and wide spectrum of bacterial virulence factors present either attached to the cell wall or extracellular virulence factors capable of producing massive tissue damage and blood dissemination of the infection [3]. The regulation of the virulence factors expression is coordinated by quorum sensing (QS), an intercellular communication system based on cell density dependent molecules with autoinductory properties that play a pivotal role in the pathogenesis of various infections. $P$. aeruginosa produce two types of quorumsensing signaling molecules (QSSMs): $N$-acylhomoserine lactones (AHL) and 2-alkyl-4-quinolone (PQS) derivatives. The AHLs molecules described so far in $P$. aeruginosa belong at two quorum sensing (QS) systems: las and $r h l$ systems whose autoinducer (AI) molecules are $N$-(3-oxododecanoyl)homoserine lactone (OdDHL, 3-oxo-C12-HSL), and $N$-butyryl-Lhomoserine lactone (C4-HSL), respectively. AHL systems are interconnected by a third mechanism that uses signaling molecules such as 2-alkyl-4-quinolone (AQ), the most relevant one being 3-hydroxy-4-quinolone (PQS = Pseudomonas quinolone signal) and its immediate precursor 2-heptyl-4-quinolone (HHQ) [4,5]. QS systems modulate numerous microbial virulence features, such as bacterial adherence and biofilm formation. It was proved that QS signaling plays an important role in cell attachment, in the differentiation of Pseudomonas biofilms and even biofilm survival [6]. The las system is indispensable for the development of a normal biofilm, including the differentiation of biofilm-like structures (such as "mushrooms" and "columns") observed in biofilms developed in vitro [7]. Some studies have shown that QS-deficient mutant strains initially form biofilms with the same dense structure as wild strains. The mutant strains produce even "mushroom"-like structures similar to wild strains that have as carbon source, glucose, but compared to wild type, mature biofilms (10 or more days of development) produced by QS deficient strains differ in size and stability of the structure, being more flexible due to the production of QS-regulated extracellular DNA [8], which acts as a stabilizer of three-dimensional biofilm structure [8]. In patients with cystic fibrosis, the growth of bacteria in biofilm determines aggravation of the disease. Recent studies have found that the QS molecules interact with eukaryotic cells and modulate also host immune response [9]. But, although the roles of the $\mathrm{N}$-acylhomoserine lactones in the modulation of immune response have been well studied, the results obtained until now were contradictory, depending on the cell line, the concentration and the time of action [10]. Moreover, the effects of AQs and other Pseudomonas molecules on the modulation of immune responses are poorly understood. The elucidation of the intimate QSSM mechanisms could contribute to the development of new anti-QS therapeutic strategies against $P$. aeruginosa infections, which are very difficult to treat.

The vascular endothelium is crucial for cell and tissue homeostasis and regulation of inflammatory response. The loss of its integrity causes plasma, proteins and cells to build up in the interstitial space, resulting in inflammation [11]. In order to avoid both chronic and acute inflammatory disease, e.g., atherosclerosis or loss of vascular volume, such as in septic shock, the soundness of the vascular permeability barrier is essential. The barrier function of the endothelium is strictly controlled by intercellular adherence junctions (AJ) and tight junctions (TJ), interconnected with cytoskeletal proteins. 3O-C12-HSL induces breaks in the epithelial barrier, disrupting cell junction and enhanced permeability by alterations in the phosphorylation status of TJ and AJ proteins [12]. The transmembrane protein vascular endothelial cadherin (VE-cadherin) is the major structural component of endothelial AJ with a pivotal role in endothelial barrier integrity. LasB protease released by $P$. aeruginosa during infection determines VE-cadherin cleavage and 
facilitates type III secretion system toxicity in endothelial cells [13]. ICAM-1 (intercellular adhesion molecule 1) and PECAM-1 (platelet endothelial cell adhesion molecule 1) are endothelial- and leukocyte-associated transmembrane proteins that permit transmigration of leukocytes into tissues and are induced by interleukins (IL-1, IL-8), tumor necrosis factor (TNF), nitric oxide (NO), and other inflammatory and stress factors. The investigations of the role of QSSM produced by $P$. aeruginosa on vascular endothelial cells (EC) are poorly investigated. The aim of this study was to evaluate the expression of adhesion and inflammatory molecules in endothelial cells treated with $P$. aeruginosa quorum-sensing (QS) molecules, in order to elucidate their role in the occurrence of tissue damages, in which endothelial cells are involved, such as: wound healing, transepithelial migration of neutrophils, lung inflammation and permeability. Also, we highlighted the impact of QSSMs on microbial attachment at inert and cellular substrata and biofilm formation in vitro.

\section{Results and Discussion Adherence and biofilm development}

Even though there are numerous recent studies about the influence of bacteria QSSMs on host cells, their results are chiefly about the effects of AHL molecules, whereas the interactions between host cells and PQS have remained largely unknown. As far as we know, this is the first publication showing how both $P$. aeruginosa-derived AHL and PQS impact adhesion and inflammatory parameters of endothelial cells. Also, the impact of the main P. aeruginosa QSSMs on key bacterial virulence factors such as attachment and biofilm formation was shown, as these behaviors are very important for the progression of the infectious process and represent the main host-pathogen interactions. Bacterial adherence to inert substrata and biofilms developed at different periods of time ( $24 \mathrm{~h}, 48 \mathrm{~h}$ and $72 \mathrm{~h}$ ) were decreased in the presence of all tested QSSMs in PAO1 culture (Figure 1). Adherence to inert substratum was decreased with $37.4 \%$ for OdDHL, $32.2 \%$ for C4HSL, 32.4\% for PQS and with $60 \%$ for HHQ compared with PAO1 control (Figure 1a). Biofilm formation was slightly inhibited by the presence of most of the tested OSSMs; only HHQ slightly increased the development of biofilm (21.7\%) at 72 hours (Figure 1b).

The inhibitory effect of adherence was also observed for cellular substrate adherence. The adherence index of PAO1 strain to endothelial cells was decreased in the presence of $20 \mu \mathrm{M}$ QSSMs compared to untreated control. The inhibitory effect was observed in the case of OdDHL (40\% inhibition) and C4HSL (30\% inhibition), and also a low inhibition (10\%) was observed in the case of PQS (Figure 2). HHQ had no significant effect on the adherence index of $P$. aeruginosa to host endothelial cells, but a change in the adherence pattern of bacteria
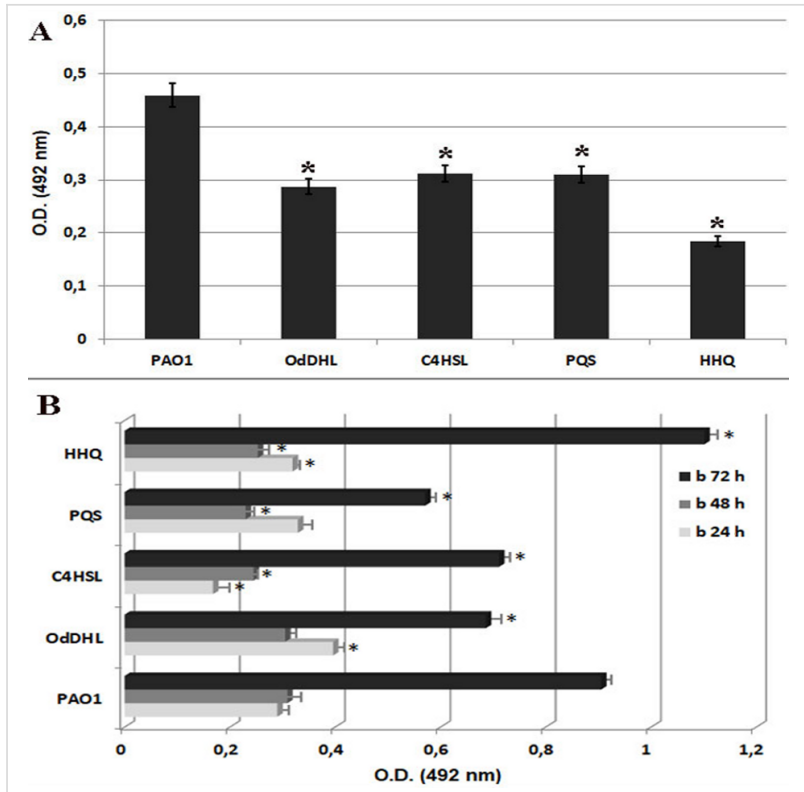

Figure 1: Graphic representation of the bacterial adherence to inert substrata (A) and biofilms developed after $24 \mathrm{~h}, 48 \mathrm{~h}$ and $72 \mathrm{~h}$ of incubation (B) in the presence of $20 \mu \mathrm{M}$ purified QSSMs $\left({ }^{*} P<0.05\right.$, based on ANOVA and Bonferroni post test of medians of 3 independent experiments performed in triplicate).

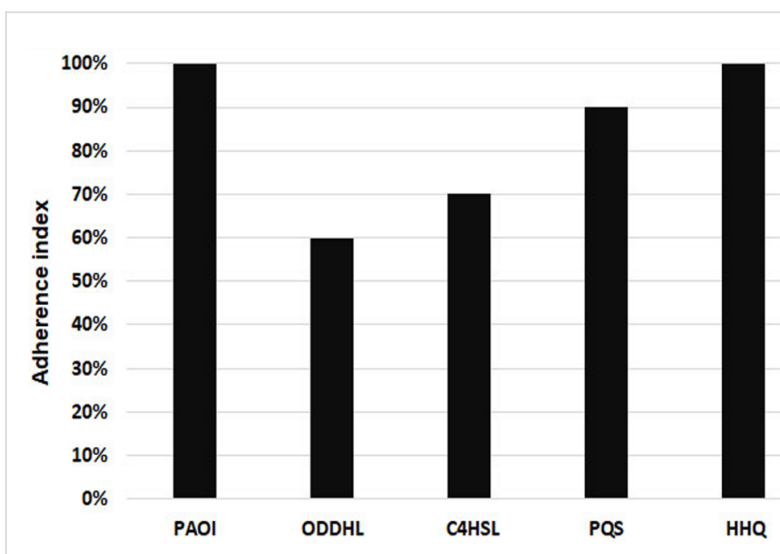

Figure 2: Graphic representation of the adherence index to cellular substrata of PAO1 strain treated with $20 \mu \mathrm{M}$ of tested purified QSSMs.

to the endothelial cells was registered, from aggregative (control PAO1) to diffuse aggregative. As seen in Table 1, changes in the adherence pattern were observed in the presence of all tested QSSMs. The main tendency of the tested QSSMs is to disperse microbial aggregates attached on the host cells, since it is clearly seen that the untreated PAO1 control has a higher aggregative potential, as compared to all other samples (Figure 3).

Flow cytometry analysis of endothelial cells adhesion molecules revealed that ICAM-1 and P-selectin production increased for all QSSMs treated samples, as compared with 
Table 1: The adherence patterns to cellular substrate of PAO1 strain treated with $20 \mu \mathrm{M}$ purified QQSMs.

\begin{tabular}{ll} 
sample & adherence pattern \\
\hline control PAO1 & aggregative \\
OdDHL & diffuse aggregative \\
C4HSL & localized aggregative \\
PQS & diffuse aggregative \\
HHQ & diffuse aggregative
\end{tabular}

control cells, the highest effect being observed in case of OdDHL and PQS. ICAM-1 showed an increased expression in endothelial cells treated with OdDHL $(75.6 \% \pm 0.48)$, C4HSL $(21.7 \% \pm 0.04)$, PQS $(43.2 \% \pm 0.011)$, HHQ $(52.1 \pm 0.019)$, as compared to control cells $(13.7 \% \pm 0.50)$. P-selectin expression was also increased for OdDHL $(27.5 \% \pm 0.03)$ and PQS $(42.2 \% \pm 0.04)$ treated cells, while C4HSL $(6.5 \% \pm 0.28)$ and HHQ $(4.4 \% \pm 0.02)$ treated samples provided lower values, comparable to untreated control cells $(10 \% \pm 0.19)$. The expression of PECAM-1 and VE-cadherin, were strongly positive for all QSSMs treated cells, as they are in untreated control endothelial cells (Figure 4). These results suggest an inflammatory/ reactive response of endothelial cells, explained by the stimula- tion of ICAM-1 and P-selectin expression which correlate with an increased production of inflammatory cytokines (such as IL-1, IL-8, TNF $\alpha$ ). These data are confirmed by recent literature showing the immunomodulatory effects of OdDHL and PQS signaling molecules [14]. It has been shown that OdDHL determines the overexpression of mRNA for IL-8 and stimulates the production of numerous active cytokines in fibroblasts and human epithelial cells in vitro. High concentrations $(100 \mu \mathrm{M})$ of purified OdDHL activate the p42/44 MAPK signaling pathway and subsequently the NF $\kappa \mathrm{B}$ transcription factor that stimulates the production of IL-8 proinflammatory cytokines [15] in human lung or fibroblast bronchial cells. 3-Oxo-C12-AHL also activates extracellular signal-regulated kinases (ERKs), which subsequently induces the activation of $\mathrm{NF} \kappa \mathrm{B}$ transcription factor. Thus, activation of NFאB is essential for maximal IL-8 production following stimulation of 3-oxo-C12-AHL cells [16]. In addition, the same group later demonstrated that $P$. aeruginosa can induce stimulation of prostaglandin E2 (PGE2) production via the COX-2 cyclooxygenase pathway. In vitro studies on human lung fibroblasts have shown that OdDHL causes overexpression of cox-2 but not cox -1 , and this effect appears to be related to NFאB activation. These autoinducers (AIs) stimulate the production of mem-

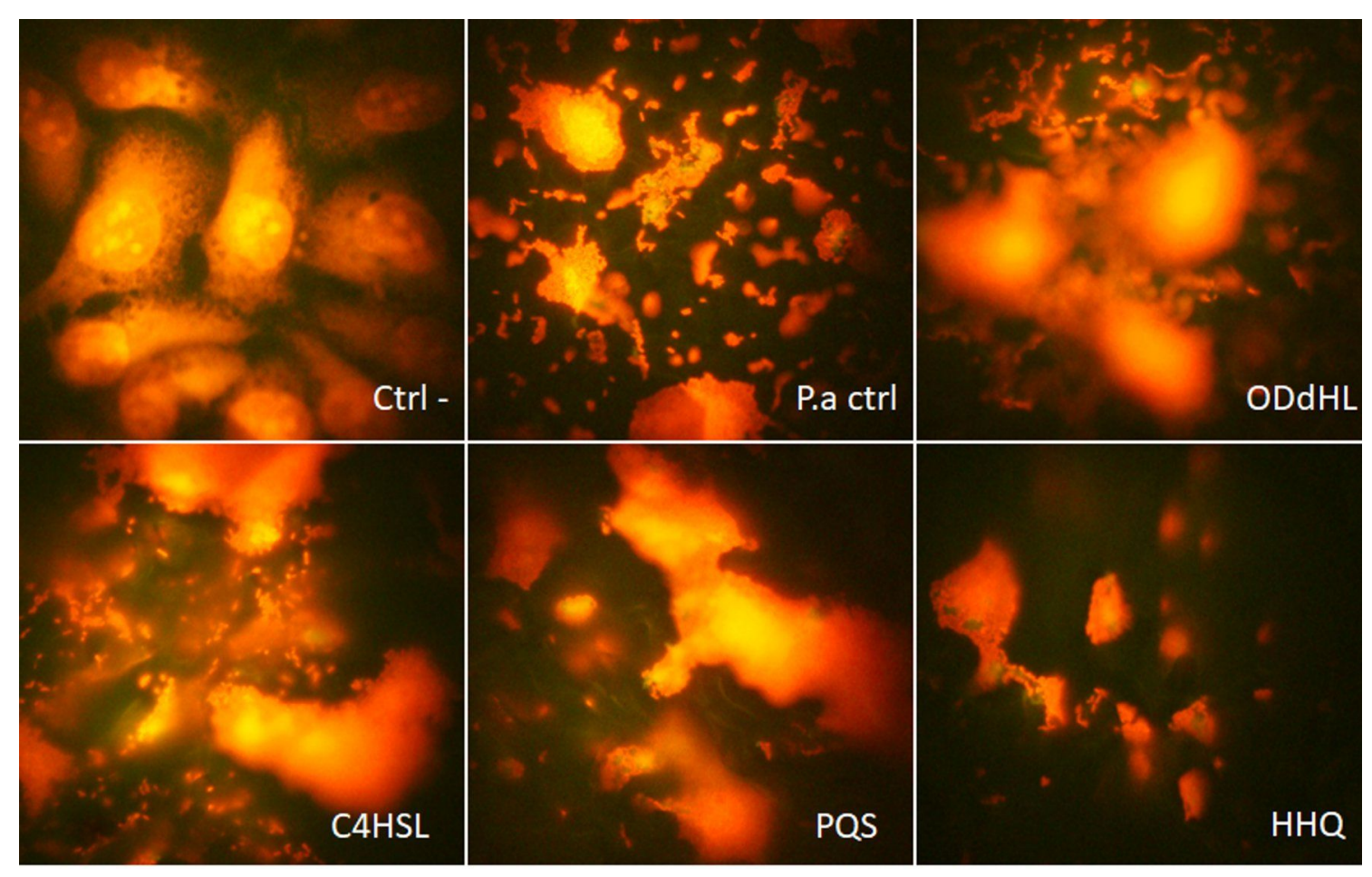

Figure 3: Fluorescence microscopy images of QSSMs treated euckariote cells, revealing attachment patterns. (Ctrl- = untreated control cells, $\mathrm{P}$.a ctrl $=P$. aeruginosa control treated cells, $\mathrm{ODdHL}=P$. aeruginosa + OddHL treated cells, $\mathrm{C} 4 \mathrm{HSL}=P$. aeruginosa $+\mathrm{C} 4 \mathrm{HSL}$ treated cells, $\mathrm{PQS}=P$. aeruginosa $+\mathrm{PQS}$ treated cells, $\mathrm{HHQ}=P$. aeruginosa $+\mathrm{HHQ}$ treated cells, ob. $100 \times$, immersion oil. 


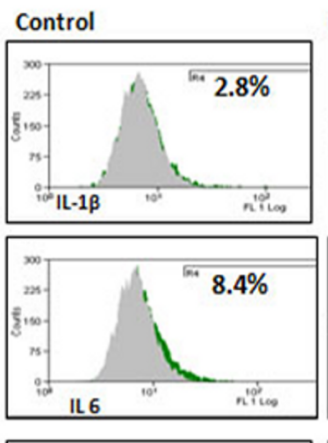

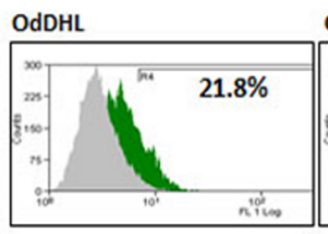

C4HSL
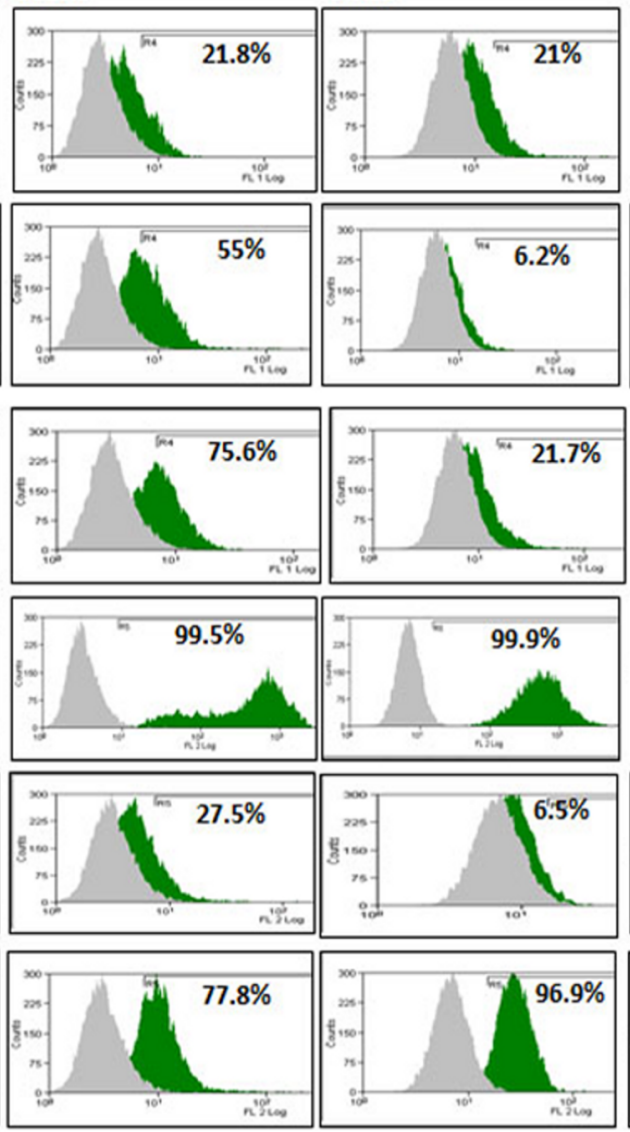
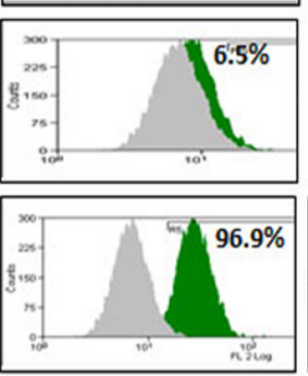
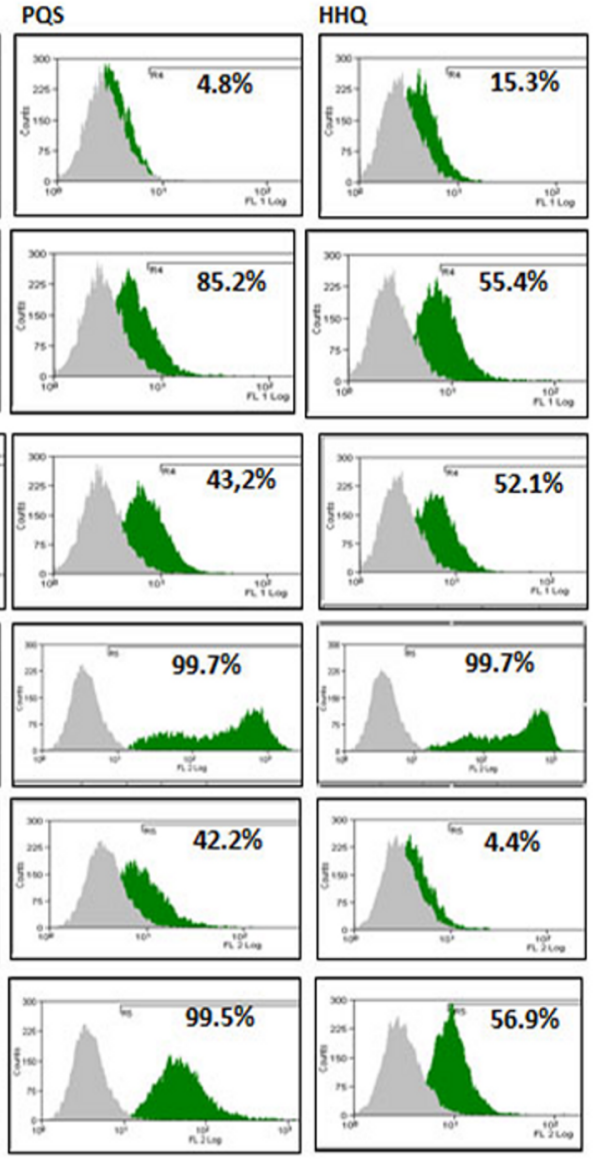

Figure 4: Flow cytometry assays for inflammatory (IL-1ß, IL-6), and adhesion markers (ICAM-1, PECAM-1, P-selectin and VE-cadherin) in endothelial cells treated with PAO1 and QSSMs $\left({ }^{*} P<0.05\right.$, Bonferroni post test of medians of 3 independent experiments performed in triplicate $(n=3)$ ).

brane-associated prostaglandin E (PGE) and PGE2 but not PGE from cytosol. It is known that PGE2 plays a role in inducing mucus secretion, vasodilatation and edema, acting as a lipid mediator in immunomodulation [17]. These results indicate that OdDHL contributes to the induction of inflammation and pulmonary pathology in $P$. aeruginosa infections, which is mainly visible in cystic fibrosis patients. PGE2 and COX-2 have been shown to play an important role in suppressing the production of reactive oxygen species by diminishing the bacterial clearance process by macrophages. For these reasons, both PGE2 and COX-2 have been proposed as future therapeutic targets for the treatment of severe pneumonia produced by $P$. aeruginosa [18].

Another signaling molecule produced by $P$. aeruginosa has been shown to have immunomodulatory effects in the host cells $[14,19]$. PQS inhibits cellular proliferation without affecting IL-2 cytokine release when T cells are activated. PQS and OdDHL significantly reduce the ability of human peripheral mononuclear cells to respond to Con-A and to anti-CD3 and anti-CD28 antibodies. PQS does not affect cell viability while OdDHL inhibits cell proliferation and viability [20]. In addition, OdDHL inhibits the release of IL-2 and TNF $\alpha$ while PQS stimulates the release of these cytokines [21]. OdDHL can control PQS production, demonstrating that both molecules, while independent, with similar or different effects, act together [22]. The expression of adhesion molecules VE-cadherin and PECAM-1 was evaluated by qRT-PCR assay, and the obtained results sustained the flow cytometry data, suggesting the ability of QSSMs to modulate adhesion of host cells. An increased expression by 9 and 11 fold, respectively, was obtained when endothelial cells were treated with C4HSL (Figure 5). Migration of leukocytes from vessels into infected tissues involves interaction with endothelium through adhesion molecules. Adhesion molecules such as integrins (Cd11/Cd18) and ICAMs are usually involved in the inflammatory process during infection. $P$. aeruginosa-activated mast cells produce IL- $1 \alpha$ and IL-1 $\beta$, which stimulate the expression of ICAM-1 and 
IL-1 $\beta$
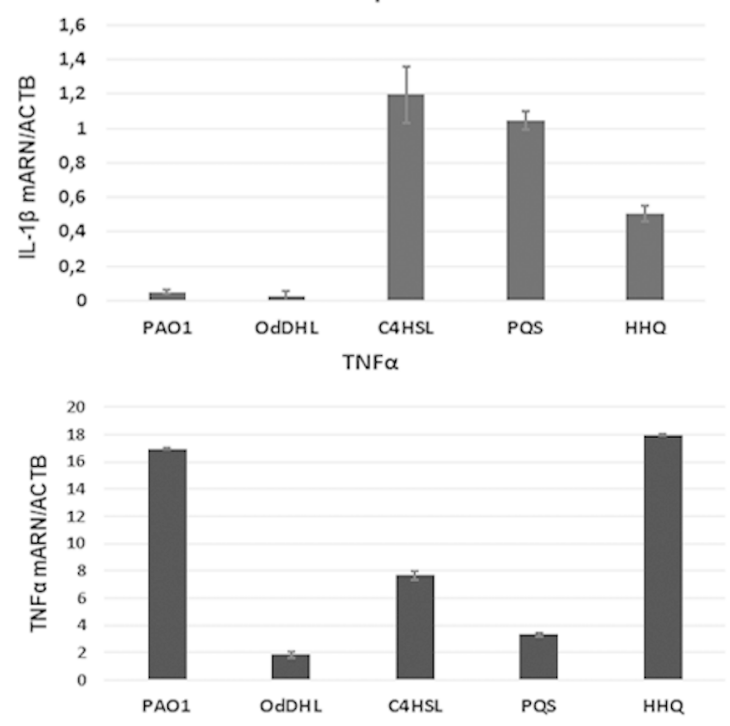

PECAM-1
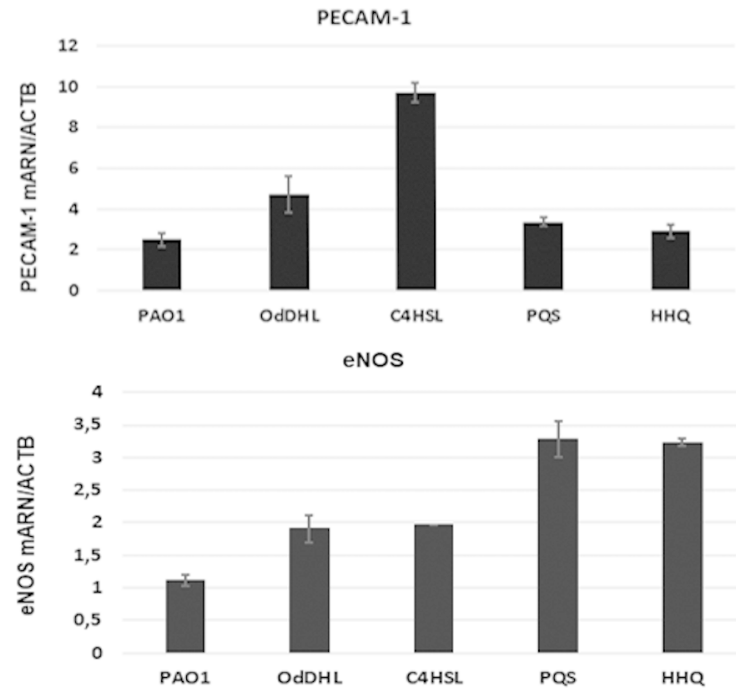

IL-6
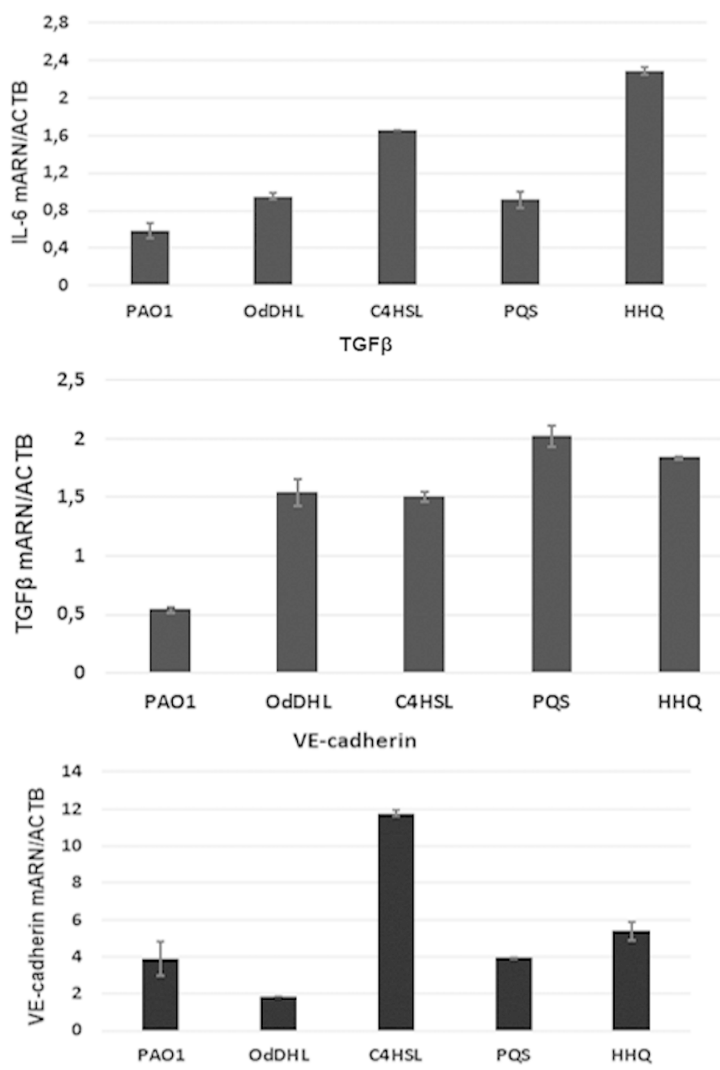

Figure 5: Expression levels (fold change) in qRT-PCR experiments for IL-1 $\beta$, IL-6, TNFa, TGF $\beta$, eNOS, VE-cadherin and PECAM-1 molecules performed on endothelial cells treated with PAO1 cultures and purified QSSMs $(P<0.05$, based on ANOVA and Bonferroni post test of medians of 4 independent experiments performed in triplicate $(n=3))$.

E-selectin in endothelial cell, this process being required for transendothelial migration [23]. Furthermore Lins et al. showed 2010 that ICAM-1 expression is modulated by ExoU cytotoxin injected via the type III secretion system, being involved in endothelial damage and bacterial dissemination [24].

\section{Inflammatory markers}

Proinflammatory cytokines and chemokines such as IL- $1 \alpha$, IL-1 $\beta$, IL- 6 and TNF $\alpha$ are produced by vascular cells such as endothelial cells and leucocytes within hours after P. aeruginosa infection. Flow cytometry assay showed elevated levels of IL- $1 \beta$ and IL- 6 . The level of IL- $1 \beta$ was increased after the stim- ulation with $20 \mu \mathrm{M}$ QSSMs. The most significant IL-1 $\beta$ expression stimulation was observed in the sample treated with OdDHL $(21.8 \% \pm 0.09)$, as well C4HSL $(21 \% \pm 0.03)$, followed by HHQ $(15.3 \% \pm 0.3)$. PQS showed no effect in the production of IL-1 $\beta$ in endothelial cells (Figure 4). The expression of IL-6 was also increased after cellular treatment with OdDHL $(55 \% \pm 0.02)$, PQS $(85.2 \% \pm 0.015)$, HHQ $(55 \% \pm 0.02)$, while C4HSL showed no significant effect in the modulation of IL-6 expression (Figure 4). QRT-PCR results showed that the presence of C4HSL, PQS and HHQ molecules increased the expression level of IL-1 $\beta$, while the PAO1 and OdDHL did not affect the level of IL-1 $\beta$. IL- 6 expression was slightly increased in the 
presence of all QSSM (0.5-2.3 fold), the differences being more significant for C4HSL and HHQ. The expression of TGF $\beta$, eNOS was significantly increased in the presence of all QSSMs, by 1.5-2.0 fold (TGF $\beta$ ) and 1.2-3.2 fold (eNOS), respectively, suggesting an activated phenotype of endothelial cells. The expression levels of adhesion molecules such as VE-cadherin and PECAM-1 were stimulated by the QSSMs, high levels being observed in the cells grown in the presence of C4HSL (Figure 5). Though there are numerous recent studies focusing on the influence of bacteria QSSMs on host cells, they are chiefly about the effects of AHL molecules, whereas the interactions between host cells and PQS have remained largely unknown. As far as we know, this is the first publication showing how both $P$. aeruginosa-derived AHL and PQS impact adhesion and inflammatory parameters of endothelial cells.

Thomas et al. in 2006 tested the ability of natural 3-oxo-C12AHL and 4 synthetic analogues to modulate cytokine production in the host cells and the results demonstrated that these soluble mediators stimulate the production of TNF $\alpha$ and nitric oxide (NO) in equine and murine macrophages [25]. The mechanism of action of QSSMs leads to the activation of NFKB signaling cascade, resulting in the upregulation of pro-inflammatory cytokines IL-6, IL-8, and TNF $\alpha$. However, our results demonstrated that TNF $\alpha$ expression is inhibited by all tested QSSMs, excepting HHQ (Figure 5). The expression of inflammatory molecules is tissue dependent and seems to be influenced by soluble virulence factors produce by $P$. aeruginosa during infection. Previous studies showed that most $P$. aeruginosa QSSMs increases the level of IL-6 in vitro. While HHQ is significantly stimulating IL-10 secretion by more than 20 -fold, PQS has no significant effect on IL-10 secretion in mesenchymal stem cells [10]. Bacterial lipopolysaccharides (LPS) and phospholipase C (PLC) induces high levels of TNF $\alpha$, IL-1 $\beta$, IFN $\gamma$, MIP-1 and MIP-2 in lung cells, but they do not affect IL-18 levels [26]. In the tracheal epithelium LPS stimulates TLR2 and TLR4, while flagellin stimulates TLR5 expression. Alveolar epithelial cells respond to $P$. aeruginosa by releasing IL-1 $\beta$, IL-6, IL-8, MIP-2 while alveolar macrophages are producing IL-1 $\beta$ and IL-18 [27].

\section{Conclusion}

This is the first article about how $P$. aeruginosa derived QSSMs influence human endothelial cell adhesion and cytokine profile. Our findings demonstrate that the major pseudomonadal AHL and PQS auto-inducers differentially modulate bacteria adherence to inert and cellular substrata and biofilm formation, but also endothelial cells adhesion and pro-inflammatory cytokine expression. These observations may help in gaining insights into understanding host-pathogen interaction and communication and may have an impact on the development of new anti-
QS therapeutic strategies to fight $P$. aeruginosa infections. Specifically, our results bring new information to help elucidating the mechanisms by which different QSSMs activate the host endothelial cells and promote epithelial lesions during P. aeruginosa infections.

\section{Experimental}

Adherence assay to inert substrate and biofilm formation was assessed by the adapted microtiter method [28]. Overnight PAO1 cultures were grown with QSSMs in 96 multi-well plates containing Tryptic Soy Broth (TSB) for $24 \mathrm{~h}, 48 \mathrm{~h}$ and $72 \mathrm{~h}$ at $37{ }^{\circ} \mathrm{C}$. After each period of time, the plates were subsequently emptied and washed three times with phosphate buffered saline (PBS). The adherent cells were then fixed with cold methanol, stained with an alkaline $1 \%$ crystal violet solution for 15 minutes, washed with distilled water and resuspended in a $33 \%$ acetic acid solution. The intensity of the suspension was spectrophotometrically assessed, the amount of adhered biomass being proportional to the absorbance value read at $492 \mathrm{~nm}$.

\section{Adherence assay to cellular substrate}

Cell culture. Endothelial cell line EA.hy926 was cultured in Dulbecco's Modified Eagle's Medium (DMEM, SigmaAldrich, St. Louis, MO, USA) containing 10\% heat inactivated fetal bovine serum (FBS), $100 \mathrm{U} / \mathrm{mL}$ penicillin, $100 \mu \mathrm{g} / \mathrm{mL}$ streptomycin, and $50 \mu \mathrm{g} / \mathrm{mL}$ neomycin. Cell cultures were maintained at $37{ }^{\circ} \mathrm{C}$ in a humidified atmosphere containing $5 \% \mathrm{CO}_{2}$ and $21 \% \mathrm{O}_{2}$. To maintain optimal culture conditions, the medium was changed twice a week. Prior the infection with P. aeruginosa and adding QSSMs the endothelial cells were cultured in a medium without antibiotics. Endothelial cells were stimulated with $P$. aeruginosa PAO1 strain (control) and purifyed QSSMs $(20 \mu \mathrm{M})(\mathrm{OdDHL}=N$-(3-oxododecanoyl)-Lhomoserine lactone; C4HSL $=N$-butyryl-L-homoserine lactone; PQS = 2-heptyl-3-hydroxy-4(1H)-quinolone; and HHQ = 2-heptyl-4-quinolone). For the adherence assay, Cravioto's adapted method was used [29]. Briefly, the endothelial cells monolayers were washed with PBS (phosphate buffered saline) and $1 \mathrm{~mL}$ of fresh medium without antibiotics was added to each well. Suspensions of $P$. aeruginosa were obtained from bacterial mid-logarithmic phase cultures grown in nutrient broth adjusted to $10^{7} \mathrm{CFU} / \mathrm{mL}$ and $1 \mathrm{~mL}$ were used for the inoculation of each well in the presence of QSSMs. The inoculated plates were incubated for two hours at $37^{\circ} \mathrm{C}$. After incubation, endothelial cells were washed three times with PBS, fixed with cold methanol and stained with $5 \mu \mathrm{g} / \mathrm{mL}$ propidium iodide. The plates were washed, dried at room temperature and examined immediately with a fluorescent microscope (Eclipse TE300, with Digital Net Camera DN100, Nikon, Tokyo, Japan), by using the $100 \times$, immersion oil objective. 
The plates were examined microscopically to evaluate the adherence index and patterns. The adherence index was expressed as the ratio between the number of the eukaryotic cells with adhered bacteria and 100 eukaryotic cells counted on the microscopic field. The adherence patterns were defined as: localized adherence (LA) when tight clusters of microorganisms were noticed on the HeLa cell surface, aggregative adherence (AA) when a microbial stacked brick pattern characterizes the attachment, diffuse adherence (DA) when the bacteria adhered diffusely, covering the whole surface of the cell.

\section{QRT-PCR assay}

The gene expression level of adhesion and inflammatory molecules in endothelial cells treated with PAO1 cultures and QSSMs, was assessed by qRT-PCR. Total cellular RNA was isolated using RNeasy Mini kit (Qiagen, Hilden, Germany) and reverse-transcription reaction was performed using M-MLV polymerase (Thermo Fisher Scientific, Massachusetts, USA). The mRNA levels were quantified by amplification of cDNA using a real-time thermocycler (Viia7 Applied Biosystems thermalcycler, Thermo Fisher Scientific, Massachusetts, USA) and TaqMan chemistry using the following assays: IL- $1 \beta$ (Hs01555410_m1), IL-6 (Hs00174131_m1), TNF $\alpha$ (Hs00174128_m1), TGF $\beta$ (Hs01086000_m1), eNOS (Hs01574665_m1), VE-cadherin (Hs00975233_m1), PECAM-1 (Hs01065279_m1), GAPDH (Hs03929097_g1). PCR reaction had the following schedule: denaturation step at $95{ }^{\circ} \mathrm{C}$ for two minutes, 40 cycles of denaturation at $95{ }^{\circ} \mathrm{C}, 15$ seconds and annealing at $60{ }^{\circ} \mathrm{C}, 40$ seconds. The genes expression level was normalized to GAPDH. The relative quantification was done using the comparative CT method and expressed as arbitrary units.

\section{Flow cytometry assay}

Endothelial cells were stimulated with $P$. aeruginosa PAO1 strain and QSSMs $(20 \mu \mathrm{M})$ for 20 hours and the expression of cell adhesion and inflammatory markers was assessed by flow cytometry (Gallios, Beckman-Coulter) using $1 \times 10^{5}$ endothelial cells stained with fluorochrome-conjugated (FITC - fluorescein-isothiocyanate and PE - phycoerythrin) primary antibodies against IL-1 $\beta$, IL-6, ICAM-1 PECAM-1, P-selectin, VE-cadherin (Beckman-Coulter). Endothelial cells were detached using trypsin (Sigma-Aldrich, USA) and washed in PBS. Cells were then incubated with the primary antibodies at room temperature in the dark for $30 \mathrm{~min}$. Further, the cells were washed twice and centrifuged at $400 \mathrm{~g}, 10 \mathrm{~min}$, in PBS suplemented with $1 \%$ BSA. For negative controls, endothelial cells were stained with the corresponding isotype-matched IgG antibodies (Beckman-Coulter). Flow cytometry data were analyzed using the Gallios software (Beckman-Coulter).

\section{Statistical analysis}

One way analysis of variance (ANOVA) was used to analyze the data (GraphPad software). Bonferroni post test was used when appropriate. $P$ values $<0.05$ were considered significant.

\section{Acknowledgements}

This work was supported by project No. 19/2018, PN-III-P11.1-PD-2016-1660, financed by the Executive Agency for Higher Education, Research, Development and Innovation Funding (UEFISCDI).

\section{ORCID ${ }^{\circledR}$ iDs}

Carmen Curutiu - https://orcid.org/0000-0002-4608-4487 Florin lordache - https://orcid.org/0000-0002-5366-6792

\section{References}

1. Farkhooy, A.; Bodegård, J.; Erikssen, J. E.; Janson, C.; Hedenström, H.; Stavem, K.; Malinovschi, A. BMC Pulm. Med. 2018, 18, No. 118. doi:10.1186/s12890-018-0655-Z

2. Buchanan, P. J.; Ernst, R. K.; Elborn, J. S.; Schock, B. Biochem. Soc. Trans. 2009, 37, 863-867. doi:10.1042/BST0370863

3. Lyczak, J. B.; Cannon, C. L.; Pier, G. B. Microbes Infect. 2000, 2, 1051-1060. doi:10.1016/S1286-4579(00)01259-4

4. Kim, K.; Kim, Y. U.; Koh, B. H.; Hwang, S. S.; Kim, S.-H.; Lépine, F.; Cho, Y.-H.; Lee, G. R. Immunology 2010, 129, 578-588. doi:10.1111/j.1365-2567.2009.03160.x

5. Williams, P.; Cámara, M. Curr. Opin. Microbiol. 2009, 12, 182-191. doi:10.1016/j.mib.2009.01.005

6. Jiménez-Gómez, P.; Pozuelo de Felipe, M. J.; Llinares Pinell, F.; García de los Ríos, J. E. Quorum-sensing in Pseudomonas aeruginosa and Salmonella: Active natural compounds as antagonists. In Communicating Current Research and Educational Topics and Trends in Applied Microbiology; Méndez-Vilas, A., Ed.; Formatex: Badajoz, Spain, 2007; pp 41-51.

7. Lazar, V. Anaerobe 2011, 17, 280-285. doi:10.1016/j.anaerobe.2011.03.023

8. Allesen-Holm, M.; Barken Bundvig, K.; Yang, L.; Klausen, M.; Webb, J. S.; Kjelleberg, S.; Molin, S.; Givskov, M.; Tolker-Nielsen, T. Mol. Microbiol. 2006, 59, 1114-1128. doi:10.1111/j.1365-2958.2005.05008.x

9. Feng, L.; Xiang, Q.; Ai, Q.; Wang, Z.; Zhang, Y.; Lu, Q. Mediators Inflammation 2016, 16, No. 4012912. doi:10.1155/2016/4012912

10. Holban, A.-M.; Bleotu, C.; Chifiriuc, M. C.; Bezirtzoglou, E.; Lazar, V. Virulence 2014, 5, 303-310. doi:10.4161/viru.27571

11. van Nieuw Amerongen, G. P. Tissue Engineering and Biomaterials; Springer: Berlin, Heidelberg, 2013; p 12.

12. Holm, A.; Vikström, E. Front. Plant Sci. 2014, 5, No. 309. doi:10.3389/fpls.2014.00309

13. Golovkine, G.; Faudry, E.; Bouillot, S.; Voulhoux, R.; Attrée, I.; Huber, P. PLoS Pathog. 2014, 10, e1003939. doi:10.1371/journal.ppat.1003939

14. Lin, J.; Cheng, J.; Wang, Y.; Shen, X. Front. Cell. Infect. Microbiol. 2018, 8, No. 230. doi:10.3389/fcimb.2018.00230 
15. Gardères, J.; Henry, J.; Bernay, B.; Ritter, A.; Zatylny-Gaudin, C.; Wiens, M.; Müller, W. E. G.; Le Pennec, G. PLoS One 2014, 9 , e97662. doi:10.1371/journal.pone.0097662

16. Smith, R. S.; Fedyk, E. R.; Springer, T. A.; Mukaida, N.; Iglewski, B. H.; Phipps, R. P. J. Immunol. 2001, 167, 366-374.

doi:10.4049/jimmunol.167.1.366

17. Smith, R. S.; Rodney, K.; Iglewski, B. H.; Phipps, R. P. J. Immunol. 2002, 169, 2636-2642. doi:10.4049/jimmunol.169.5.2636

18. Sadikot, R. T.; Zeng, H.; Azim, A. C.; Joo, M.; Dey, S. K.; Breyer, R. M.; Peeble, R. S.; Blackwell, T. S.; Christman, J. W. Eur. J. Immunol. 2007, 37, 1001-1009. doi:10.1002/eji.200636636

19. lordache, F.; Curutiu, C.; Holban, A.M.; Andrei, E.; Chifiriuc, C.; Lazar,V.; Ditu, L.M.; Constantinescu, A.; Maniu, H. e-poster presented at ECCMID 2015, topic Microbial Pathogenesis and Biofilms, Copenhagen, Denmark.

20. Skindersoe, M. E.; Zeuthen, L. H.; Brix, S.; Fink, L. N.; Lazenby, J.; Whittall, C.; Williams, P.; Diggle, S. P.; Froekiaer, H.; Cooley, M.; Givskov, M. FEMS Immunol. Med. Microbiol. 2009, 55, 335-345. doi:10.1111/j.1574-695X.2008.00533.x

21. Hooi, D. S. W.; Bycroft, B. W.; Chhabra, S. R.; Williams, P.; Pritchard, D. I. Infect. Immun. 2004, 72, 6463-6470. doi:10.1128/IAI.72.11.6463-6470.2004

22. Middleton, B.; Rodgers, H. C.; Cámara, M.; Knox, A. J.; Williams, P.; Hardman, A. FEMS Microbiol. Lett. 2002, 202, 1-7. doi:10.1111/j.1574-6968.2002.tb11019.x

23. Lin, T.-J.; Garduno, R.; Boudreau, R. T. M.; Issekutz, A. C. J. Immunol. 2002, 169, 4522-4530. doi:10.4049/jimmunol.169.8.4522

24. Lins, R. X.; de Assis, M. C.; Mallet de Lima, C. D.; Freitas, C.; Maciel Plotkowski, M. C.; Saliba, A. M. Microbes Infect. 2010, 12, 154-161. doi:10.1016/j.micinf.2009.11.005

25. Thomas, G. L.; Böhner, C. M.; Williams, H. E.; Walsh, C. M.; Ladlow, M.; Welch, M.; Bryant, C. E.; Spring, D. R. Mol. BioSyst. 2006, 2, 132-137. doi:10.1039/B517248A

26. Wieland, C. W.; Siegmund, B.; Senaldi, G.; Vasil, M. L.; Dinarello, C. A.; Fantuzzi, G. Infect. Immun. 2002, 70, 1352-1358. doi:10.1128/IAI.70.3.1352-1358.2002

27. Lin, C. K.; Kazmierczak, B. I. J. Innate Immun. 2017, 9, 250-261. doi:10.1159/000455857

28. Fleming, D.; Chahin, L.; Rumbaugh, K. Antimicrob. Agents Chemother. 2017, 61, No. e01998-16. doi:10.1128/AAC.01998-16

29. Holban, A. M.; Cotar, A. I.; Chifiriuc, M. C.; Bleotu, C.; Banu, O.; Lazar, V. Afr. J. Microbiol. Res. 2013, 7, 3453-3460.

\section{License and Terms}

This is an Open Access article under the terms of the Creative Commons Attribution License (http://creativecommons.org/licenses/by/4.0). Please note that the reuse, redistribution and reproduction in particular requires that the authors and source are credited.

The license is subject to the Beilstein Journal of Organic Chemistry terms and conditions:

(https://www.beilstein-journals.org/bjoc)

The definitive version of this article is the electronic one which can be found at:

doi:10.3762/bjoc. 14.235 